\title{
Two Cases of Actinomycosis with Variable Presentation and Outcome
}

\author{
SOLAIMAN,${ }^{1}$ KAZI MD RUBAYYATH ANOWAR, ${ }^{2}$ RUBAINA ZAMAN,${ }^{3}$ MD. RAIQ RAIHAN CHOWDHURY, ${ }^{2}$ REZAUL \\ EKRAM, ${ }^{4}$ ABUL HAYATH MANIK, ${ }^{1}$ MOHAMMAD RAFIQUL ISLAM, ${ }^{5}$ MD. ROBED AMIN ${ }^{6}$
}

\begin{abstract}
Actinomycoses, a rare bacteriological illness whichis primarily involved in cervico-facial infections in susceptible populations. Thoracic, abdominal, pulmonary and cardiac involvement are also reported but are exceedingly rare in incidence. So, high degree of suspicion is required for early detection and treatment. We confirmed 2 cases of Actinomycosis, in the period of January, 2014 to July, 2015 with variable presentation and different outcome.
\end{abstract}

Key words: Actinomycosis, Cervicofacial, Disseminated

\section{Introduction:}

Actinomyces israelii are normal commensals of the oropharynx, gastrointestinal tract and female urogenital tract. They are typically found in carious teeth, dental plaque, and gingival and tonsillar crypts. ${ }^{1}$ As such, the bacteria do not cause an infection unless there is a break in the mucosa or skin, as can occur with dental sepsis, appendicitis, aspiration, trauma, surgery, etc.Actinomycosisa multisystem disease, which usually involve cervico-facial area, but may have a rare dissemination course with poor outcome. Incidence is two to four times greater in males compared to females, possibly due to higher incidence of facial trauma and poorer oral hygiene in males. It can be misdiagnosed as TB, neoplasia, and necrotizing pneumonia.

Actinomycoses, a rare bacteriological illness whichis primarily involved in cervico-facial infections in susceptible populations. Thoracic, abdominal, pulmonary and cardiac involvement are also reported but are exceedingly rare in incidence. ${ }^{2,3}$

1. Indoor Medical Officer, Department of Medicine, Dhaka Medical College

2. Honorary Medical Officer, Department of Medicine, Dhaka Medical College

3. Resident, Altoona Medical College, USA

4. Assistant Registrar, Department of Medicine, Dhaka Medical College

5. Assistant Professor, Department of Medicine, Dhaka Medical College

6. Associate Professor, Department of Medicine, Dhaka Medical College

Address of correspondence: Dr Md. Robed Amin, Associate Professor, Department of Medicine, Dhaka Medical College and Hospital. Email: robedamin@yahoo.com. Mob:01711725787

\section{Case 1:}

A 30-years pregnant female who presented with pain, itching around maxillary territory for 5 months. There was history of occasional whitish discharge from the right side of her face for last three years. Pain was localized over the upper part of the right side of her face. That wasshooting or shock-like in nature and was associated with itching. She had no history of similar disease in any other part of her body. she also had no other skin conditions, hypopigmented or hyperpigmented areas, prolonged fever, genital ulceration or genital discharge. Earlier she was treated with antitubercular chemotherapy for 2 occasions without significant improvement. But the discharge from the lesion stopped permanently but her other symptoms persisted.

About one year later she developed another episode of similar symptoms. Her past history of illness was unremarkable other than her involvement in a road traffic accident in 2010. In that event, she received trauma to the upper part of the right side of her face and around her right eyebrow.

On general examination, her vital parameters were within normal range and there was no significant abnormality apart from multiple disfiguring scars over the upper part of the right side of her face. Local examination revealed there were multiple swelling of variable size and shape with an opening at the superolateral end,without any discharge(fig 1). The overlying skin was free and was slightly tender on palpation. Few of the swellings were mobile while others are not. There was no temperature or colour change, no cervical lymphadenopathy, no impairment of sensation and no weakness of muscles of mastication or muscles of facial expression. There was no tenderness over the jaw bones. Examination of the oral cavity also revealed no visible lesion. 


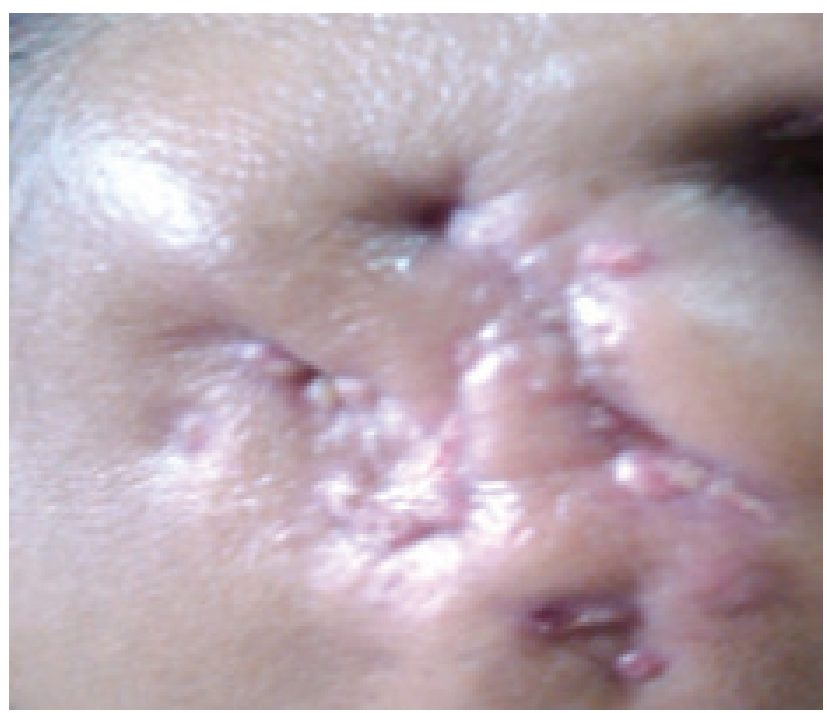

Fig.-1: Multiple swelling with opening and pitting skin

On March 02 in 2013 her ESR was $34 \mathrm{~mm}$ in $1^{\text {st }}$ hour,urine RME showed Pus cells: 1-3 /HPF with few epithelial cell.FNAC of facial swelling revealed many neutrophils and mixed with histiocytes consists of acute on chronic inflammation with tuberculosis may be a possibility. MT was $02 \mathrm{~mm}$ with induration. On $10^{\text {th }}$ March 2013 histopathology of facial skin lesion revealed. Sections from the submitted tissue show ulcerated skin with subcutaneous fatty \&fibromuscular tissue of face lined by hyperkeratotic, parakaratotic, \& marked acanthotic squamous epithelium with areas of haemorrhage, ulceration and necrosis of surface lining epithelium consisting with Benign chronic nonspecific inflamed \& ulcerated lesion.

During hospital stay at $\mathrm{DMCH}$, Investigations revealed. Complete blood count with $\mathrm{Hb}: 10 \mathrm{gm} / \mathrm{dL}, \mathrm{RBC}: 3.03 \times 10^{12}$ / L, WBC: $6.6 \times 10^{9} / \mathrm{L}$, Montoux Test was $02 \mathrm{~mm}$ with induration.Plain Xray face A-P and lateral view suspected fracture is seen in the ramus of right mandible and mild soft tissue. Her urine for pregnancy test was positive. HIV $(1 \&$ 2) was negative.Ultasonography of whole abdomen showed early pregnancy with all other normal findings. Skin scrapping for fungus was negative. CT scan of face with 3D reconstruction revealed maxillary involvement of actinomycosis.

No bacterial growth was found at skin lesion culture at $37^{0}$ $\mathrm{C}$ in aerobic condition after 48 hours incubation. Skin biopsy for histopathology revealed ulceration of surface with dermis showing chronic abscess with polymorphs, surrounding granulation tissue and fibrosis. Inside the inflammatory infiltrate are occasional matted colonies of bacteria forming

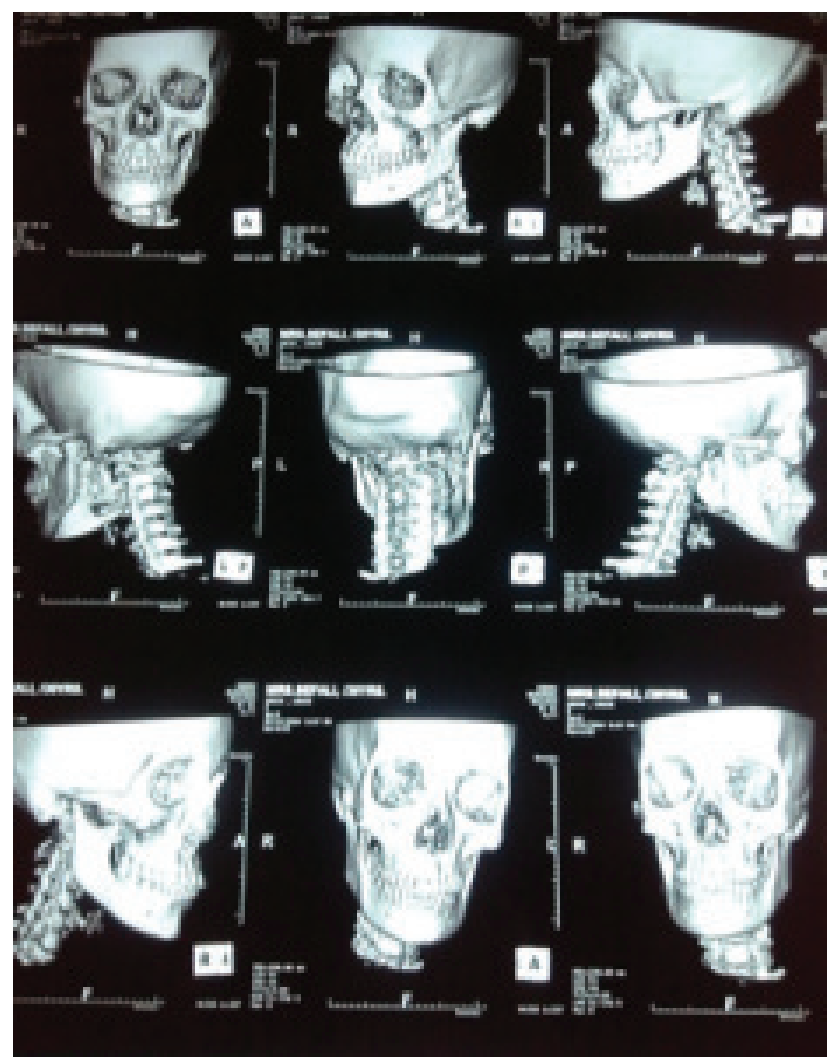

Fig 2: CT scan of face revealed maxillary involvement ofactinomycosis

grain consistent with Actinomycosis. We started the treatment with Penicillin IV until completion of pregnancy. She decided for medical termination of pregnancy. The response to penicillin was inadequate and she developed discharging pus and a new swelling at right supraorbital margin. The polymicrobial superinfection with Actinomycosis was diagnosed and she was put on Amoxycillin and clavulanic acid and Doxicycline for 6 months. She completed the course and became afebrile and all lesions were healed except the scarring. She was advised for total one year duration of treatment and subsequent evaluation of plastic surgeon for reconstructive surgery in face.

\section{Case 2:}

A 15 year male admitted with a slowly progressive skin lesion on anterior and posterior chest wall.These lesions transformed to pustules and discharged purulent, nonsanguineous fluid. He had history of jaundice, recurrent fever and weight loss for the past four years. Since the onset of the pustules four years ago, he has received different group antibiotics. Approximately four months prior to his admission, while undergoing homeopathic treatment, the patient developed acute lower extremity edema, jaundice and abdominal pain with distension. Upon presentation, he 
denied cough, dyspnea, chest pain, hemoptysis and other pulmonary findings.

On examination he was ill looking with below average nutrition, His temperature was $102{ }^{0} \mathrm{~F}$, pulse- $100 \mathrm{~b} / \mathrm{m}$, Blood Pressure was 90/60 $\mathrm{mm}$ of $\mathrm{Hg}$. There was a restricted lumbar movement due to pain. He was anemic, cachexic, icteric. There was bipetalpitting edema with diffuse abdominal tenderness with ascites. There were no stigmata of chronic liver disease. Respiratory system examination revealed features of consolidation. Other systemic examinations were normal.

There was a diffuse patch of extensive tender nodules with purulent discharge over the anterior, right thoracic region, extending to the back and $4 \mathrm{~cm}$ above the umbilicus. (Fig 3 ). The older crop of pustules had ulcerated and skin fibrosis

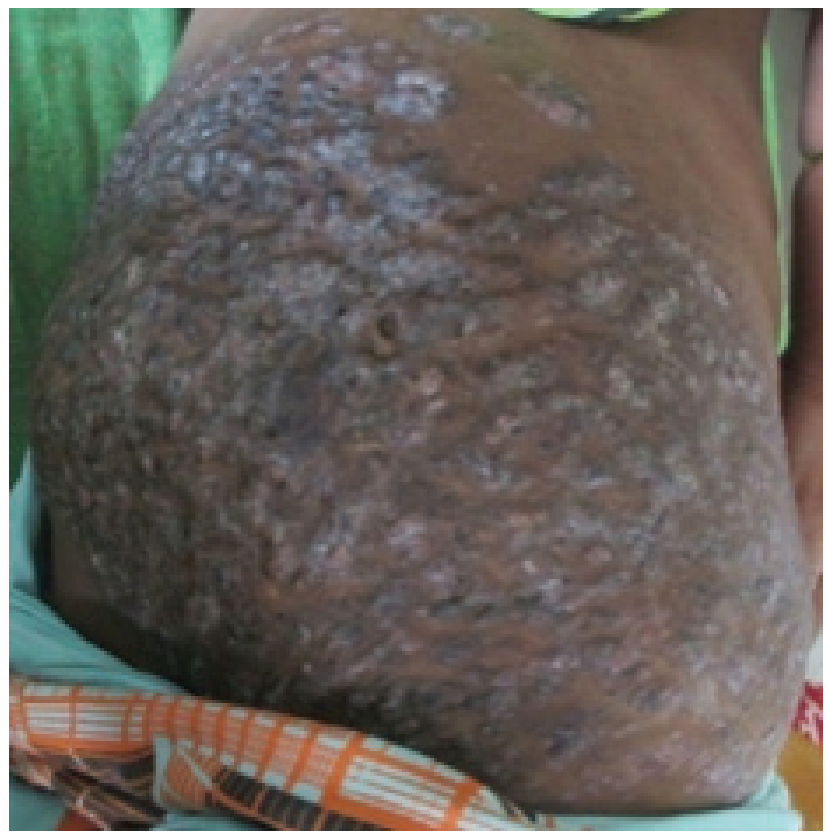

Fig 3:Skin lesions in abdomen with nodule and pustules and fibrosis was visible. Mild palpation and pressure reproduced pain and scant purulent discharge was noted from the newer, peripheral lesions. A few crops of similar pustular lesions were also observed in bilateral shin area.

Investigations of Complete Blood Count with peripheral blood film (PBF) showed $\mathrm{Hb} \%: 9.8 \mathrm{mg} / \mathrm{dL}$, Total count of white blood cell- $23 \times 10^{\wedge} 3 / \mathrm{uL}$ with neutrophilia of $85 \%$, lymphocytes $8 \%$. ESR was $20 \mathrm{~mm}$ in $1^{\text {sthour. Red cell }}$ distribution width was elevated at $20 \%$.PBF showed microcytic, hypochromic anemia. The progression of the Complete Blood Count and Liver Function Test are tabulated below.

Basic metabolic panel was significant for potassium of 2.9 and albumin of 15 (normal 35-50 g/L).ProthombinTime was18.99 second with INR 1.59. Urine routine microscopic examination showed trace albumin, no glucose, and was otherwise within normal limits.Ascitic fluid study showed WBC count of 150/uL, 40\% PMN, 60\% lymphocyte and few RBCbut no acid fast bacilli and pus cells. Gram stain was also negative for organisms. USG of W/A showed extensive free fluid collection in the peritoneal cavity, with the liver pushed caudally. Liver span is $7 \mathrm{~cm}$. Liver parenchyma was coarse and nonhomogeneous with no focal lesions present. Spleen was $12.6 \mathrm{~cm}$. Parenchyma of both kidneys was echogenic with poor cortico-medullary differentiation. Gall bladder was thick-walled and contracted.Fibroscan showed a fibrosis score $25.7 \mathrm{kPA}(>9.3$ is indicative of fibrosis and $>12.3$ indicates cirrhosis) \& was diagnosed with stage F4 cirrhosis. A 24 hour urine copper: 53ug (normal 20-50 ug). Upper GI endoscopy was normal with no esophageal or gastric varices seen.ANA was found to be negative. Blood culture was negative. Patient's sputum culture grew Enterobacter sensitive to Imipenem, amikacin and cefepime. CT scan of chest and upper abdomen showed communicating lesions of lung \& chest wall.

Table-I

Basic investigations done in second case

\begin{tabular}{lccc}
\hline & Nov 12, 20143 & Dec 15, 2014 & Dec 31, 2014 \\
\hline Hemoglobin (mg/dl) & 9.8 & 8.4 & 8.3 \\
WBC $\left(10^{\wedge} 3 / \mathrm{uL}\right)$ & 23 & 21.31 & 20.7 \\
Neutrophils (\%) & 85 & - & 74.2 \\
Lymphocytes (\%) & 8 & - & 15.9 \\
Platelet (10^9/L) & 230 & 320 & 537 \\
Total bilirubin (mg/dL) & 8.62 & 2.42 & 1.39 \\
ALT (IU/L) & 91 & 295 & - \\
AST (IU/L) & 30 & 36 & - \\
Alkaline phosphate (IU/L) & 50 & - \\
\hline
\end{tabular}



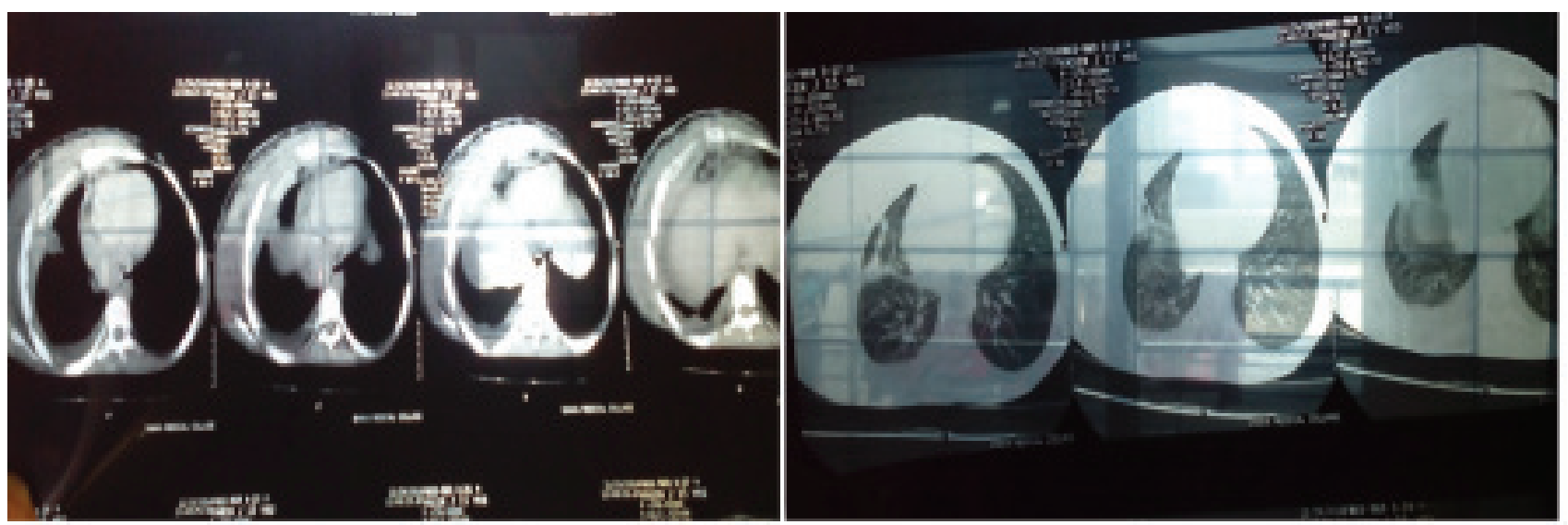

Fig 5: CT chest revealed communicating lesions of lung and chest wall.

A skin biopsy in November of 2014 showed abscess cavity in the dermis lined by granulation tissue. Abscess contained colonies of actinomycetes surrounded by suppuration. A third and final biopsy of the dermis at different site on the thorax showed sinus tract with granulation, dense acute and chronic inflammatory cells. No granuloma or malignancy was seen. The sequential involvement of pulmonary, chest wall, skin, and subdiaphramatic extension leading to liver involvement with repeated biopsy of actinomycosicolony demonstrated disseminated actinomycosis as our anatomical diagnosis.Patient was started on Imipenem $500 \mathrm{mg}$ intravenously 6 hourly for enterobacter grown on sputum culture. Clindamycin was started at $300 \mathrm{mg}$ per oral (PO) twice daily and Amoxicillin-clavulinic acid $1.2 \mathrm{~g}$ Intravenously (IV)thrice daily for treatment of diffuse thoracic actinomycosis with chest wall infiltration.Furosemide $40 \mathrm{mg}$ and Spironolactone $50 \mathrm{mg}$ was given for lower extremity edema and ascites, which improved over time. Pruritus was treated with chlorphenamine and hydroxyzine per oral.

Over the next 6 weeks, during which the patient received IV Amoxicillin-clavulinic acid and PO Clindamycin, the dissemination of the pustules stopped and the patient's jaundice resolved. The neutrophil count also improved, although total white count was at 20,000 two months after initiation of IV antibiotics. The patient continued to have occasional fevers six weeks after initiation of IV Amoxicillinclavulanic acid, with a maximum temperature of 103 degrees. Patient remained in the hospital under close observation with the plan to switch to PO penicillin-based antibiotics after 2 months of IV antibiotics. During course of the treatment there was flactuiation of response, jaundice progressively increased and he developed progressing respiratory failure. He was transferred to ICU. Despite all efforts, the patient expired due to multi organ failure.

\section{Discussion:}

The cervicofacial actinomycoses can present either as an acute, usually odontogenic, abscess or cellulitis of the floor of the mouth, or as a slowly developing chronic hard painless reddish or livid swelling. ${ }^{3,4}$ The primarily chronic formis characterized by regression and cicatrization of central suppurative foci. Infection progresses peripherally producing hard painless livid infiltrations. High index of suspicion is crucial to diagnose when there is no sulphar granule discharge. In this index case although the diagnosis was not established early and patient also received antitubercular chemotharpy for 6 months, she did not have extension of disease toward cranial cavity or mediastinum. The neutrophil predominance in the necrosed area can be a differentiating histopathology from tuberculosis in this case as granulation and fibrosis can develop in any chronic process. ${ }^{5}$

The chronicity and the extension of disease process can lead to false diagnosis of tuberculosis and malignancy in many cases. The anatomical location, the polymorph involvement, the sulphar granules and extensive ulcer with fibrosis is the hallmark to diagnose actinomycosis. Treatment is rewarding if lesions remain localized and does not involve the cranial cavity. In our $1^{\text {st }}$ case after giving antibiotics for prolonged period there was much improvement of her facial lesions. The treatment outcome is good in most reported case in literature.Cervicofacial actinomycosis is an uncommon medical disorder where the diagnosis is late due to lack of strong suspicion.

After cervicofacial actinomycosis, pulmonary infection is the second most common manifestation of this disease accounting for $15-45 \%$ of all cases ${ }^{5}$ with encroachment into surrounding tissue and formation of masses that can mimic malignancy. ${ }^{5}$ Other than the lungs, thoracic actinomycosismay also involve the pleura, mediastinum, 
chest wall and pericardium. ${ }^{6}$ The infection can have transdiaphragmatic or rarely, hematogenous dissemination. In thoracic actinomycosis, there can be formation of abscesses in the lung parenchyma, with surrounding pleural thickening, rib involvement and drainage into the skin through sinus tracts. The second case of thoracic actinomycosis that has extended transthoracically and trans diaphragmatically and also disseminate haematogenously and ultimately involving the liver, skin and lower extremities, causing extensive multi-system damage and morbidity. The average duration of illness before definitive diagnosis is six months. ${ }^{7}$ In our patient, his first chest wall biopsy was in July, 2011 (within 6 months of his initial presentation), which showed granulation tissue, chronic and acute inflammation, and sulfur granules of actinomyces colonies. Due to inappropriate choice of antibiotics, the treatment failure was observed and the recurrences of disease become extensive.The patient continued to have progressive local and possibly trans-diaphragmatic spread of the disease, causing cirrhosis and jaundice. ESR was found to be elevated, along with predominant neutrophilia. CT of the chest showed Right lower pleural, parenchymal, rib and chest wall infiltration.

Diagnosis thus requires a combination of biopsy, imaging, lab work and physical findings. The liver involvement was worked out for active exclusion of common causes like infection, autoimmune and metabolic. Although liver involvement is commonly a suppurative process in actinomycosis, the chronicity of illness can lead to fibrosis and hence the histopathology may mimic cirrhosis. ${ }^{5}$ Due to extensive suppuration in skin, the liver biopsy could not be performed. But fibroscan revealed F4 cirrhosis. This indicates the chronic involvement of liver in this disseminated case with partial treatment and extensive fibrosis by actinomycosis. We were unable to obtain a culture of the lesions owing to the strict lab protocols involved in growing actinomycosis in an anaerobic environment.

Recommended treatment with clinically proven efficacy include IV penicillin G $150,000 \mathrm{U} / \mathrm{kg} /$ day to $200,000 \mathrm{U} / \mathrm{kg} /$ day divided TID or QID for 4-6 weeks. This is followed by a prolonged $\mathrm{PO}$ penicillin $\mathrm{V} 2 \mathrm{~g}$ /day to $4 \mathrm{~g}$ /day course divided three to four times daily for an additional six to twelve months. The Tetracycline/ doxycycline, erythromycin and clindamycin may also be used if there is a penicillin allergy. ${ }^{7}$ The selection of antibiotics in our case was a combination of amoxicillin-clavulanic acid and clindamycin, both shown to have clinical efficacy and success in clinical trials in the past and the choice was taken due to unavailability of penicillin in national market. The duration of antimicrobials may be further prolonged if infection is extensive or in the presence of dissemination at different places. Surgery remains an important therapeutic adjunct with antibiotics and may be required if complications are present, including well-defined abscess, empyema, life-threatening hemoptysis or in blocked discharging fistulas that need surgical opening. ${ }^{8}$ Even with surgical debulking of the mass, it is important to continue prolonged antibiotic therapy, as surgery alone is usually not curative and inadequate antibiotic therapy may result in fistulas and empyema. Prognosis of disseminated actinomycosis involving pulmonary and hepatic tissue is usually less favorable compared to the cervicofacial and abdominal forms. With greater dissemination, transdiaphragmatic or hematological spread, the prognosis secondary to delayed diagnosis and/or treatment, the prognosis is even poorer. In our second case, surgeons were not interested to intervene due to progressive jaundice and septicaemia. The dissemination with septicaemia progressed rapidly and thus theoutcome was unfavourable.

Conclusion:High degree of suspicion is required for early detection and treatment for both localized and disseminated cases of actinomycosis.It can be misdiagnosed as TB, neoplasia, and necrotizing pneumonia. Appropriate investigation can revealearly diagnosis and appropriate treatment with adequateduration is crucial. Still the disseminated cases have poor prognosis.

\section{Conflicts of Interest: None.}

\section{References:}

1. Agents of Actinomycosis, in Principles and Practice of Infectious Diseases, 7th ed, GL Mandell et al (eds). New York, Churchill Livingstone, 2010:3209-3219.

2. Actinomycosis, in Harrisons Principles of Internal Medicine, $18^{\text {th }}$ ed. Thomas A Russo.Macgrew Hill,2014, Vol II pp-13261330

3. SchaalKP, Lee HJ (1992). Actinomycete infections in humans: a review. Gene 1992;115:201-11.

4. Pulverer G, Schütt-Gerowitt H, SchaalKP. Human cervicofacialactinomycoses: microbiological data of 1997 cases. Clin Infect Dis2003;37:490-7.

5. Acevedo, Francisco, et al. “Actinomycosis: a great pretender. Case reports of unusual presentations and a review of the literature.'International Journal of Infectious Diseases 12.4 2008:358-362.

6. Smego, R, Jr., and G. Foglia. Actinomycosis.Clin Infect Dis 1998;26:1255-61.

7. Mabeza, Macfarlane. Pulmonary actinomycosis.EurRespir J 2003;21:545-551.

8. Chernihovski et al.Chest Wall Actinomycosis. IMAJ2007;9:686-687. 